\title{
Longitudinal beam profile measurements at CTF3 using a streak camera
}

\author{
C.P. Welsch ${ }^{\text {}}$, H.H. Braun ${ }^{a}$, E. Bravin ${ }^{a}$, R. Corsini ${ }^{a}$, S. Döbert ${ }^{a}$, T. Lefèvre ${ }^{a}$, F. \\ Tecker $^{a}$, P. Urschütz ${ }^{a}$, B. Buonomo ${ }^{b}$, O. Coiro ${ }^{b}$, A. Ghigo ${ }^{b}$ and B. Preger ${ }^{b}$ \\ ${ }^{a}$ CERN, Geneva, Switzerland \\ ${ }^{b}$ INFN, Frascati, Italy \\ E-mail: Cwelsch@web.de
}

ABSTRACT: The proposed Compact Linear Collider (CLIC) is a multi-TeV electron-positron collider for particle physics based on an innovative two-beam acceleration concept. A highintensity drive beam powers the main beam of a high-frequency $(30 \mathrm{GHz})$ linac with a gradient of $150 \mathrm{MV} / \mathrm{m}$, by means of transfer structure sections. The aim of the CLIC Test Facility (CTF3) is to make exhaustive tests of the main CLIC parameters and to prove the technical feasibility. One of the points of particular interest is the demonstration of bunch train compression and combination in the Delay Loop and in the Combiner Ring. Thus, detailed knowledge about the longitudinal beam structure is of utmost importance and puts high demands on the diagnostic equipment.

Among others, measurements with a streak camera have been performed on the linac part of the CTF3 as well as on the newly installed Delay Loop. This allowed e.g. monitoring of the longitudinal structure of individual bunches, the RF combination of the beam, the behavior during phase shifts and the influence of the installed wiggler. This article first gives an overview of the CTF3 facility, then describes in detail the layout of the long optical lines required for observation of either optical transition radiation or synchrotron radiation, and finally shows first results obtained during the last machine run this year.

KEYWORDS: Beam-line instrumentation (beam position and profile monitors; beam-intensity monitors; bunch length monitors); Instrumentation for synchrotron radiation accelerators; Instrumentation for particle accelerators and storage rings - low energy (linear accelerators, cyclotrons, electrostatic accelerators).

\footnotetext{
* Corresponding author.
} 


\section{Contents}

\begin{tabular}{|ll}
\hline 1. Introduction & 1 \\
\hline
\end{tabular}

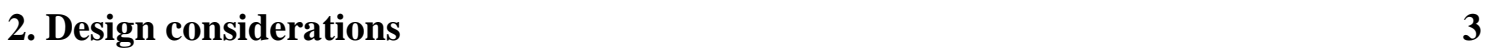

\begin{tabular}{|lr|}
\hline 3. Light characteristics from MTV0361 and MTV0550 & 4 \\
\hline 3.1 Measurements from MTV0361 using synchrotron light & 4 \\
\hline 3.2 Measurements from MTV0550 using optical transition radiation & 5 \\
\hline
\end{tabular}

\begin{tabular}{lr|}
\hline 4. ZEMAX Results & $\mathbf{6}$ \\
\hline 4.1 Layout of the optical line from MTV0361 & 6 \\
\hline 4.2 Layout of the optical line from MTV0550 & 7 \\
\hline
\end{tabular}

\begin{tabular}{|lr|}
\hline 5. Results from Measurements & $\mathbf{1 0}$ \\
\hline 5.1 Monitoring of the RF bunch combination & 10 \\
\hline 5.2 Monitoring of phase switch using sub-harmonic bunchers & 12 \\
\hline 5.3 Modification of track length with a wiggler & 12 \\
\hline 5.4 Bunch Length Measurements with a Streak Camera & 13 \\
\hline
\end{tabular}

6. Outlook

\section{Introduction}

CTF3 is being installed at CERN in the existing buildings of the LEP pre-injector accelerators LIL and EPA with the aim to demonstrate the technical feasibility of CLIC [1] [2] [3], [4]. The complex starts with a $3 \mathrm{GHz}$ linac that produces a pulsed electron beam with a present maximum energy of $150 \mathrm{MeV}$. The separation between individual bunches at the end of the linac is $20 \mathrm{~cm}$ - twice the linac RF wavelength. Moreover, the macro bunch is composed by alternated sequences $140 \mathrm{~ns}$ long of even and odd buckets, with the difference in phase between them being one RF wavelength.

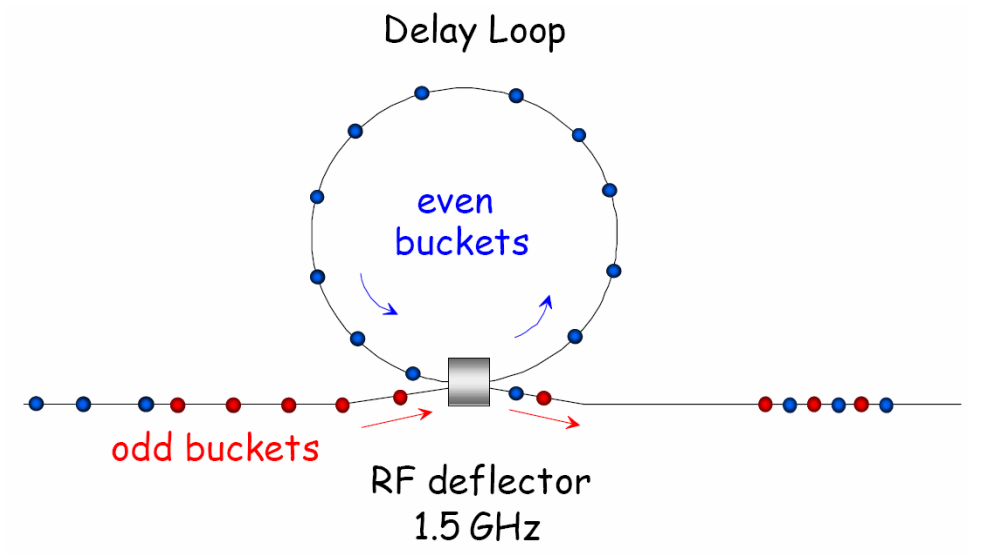

Figure 1 Schematic drawing of the injection scheme into the CTF3 Delay Loop [2]. 


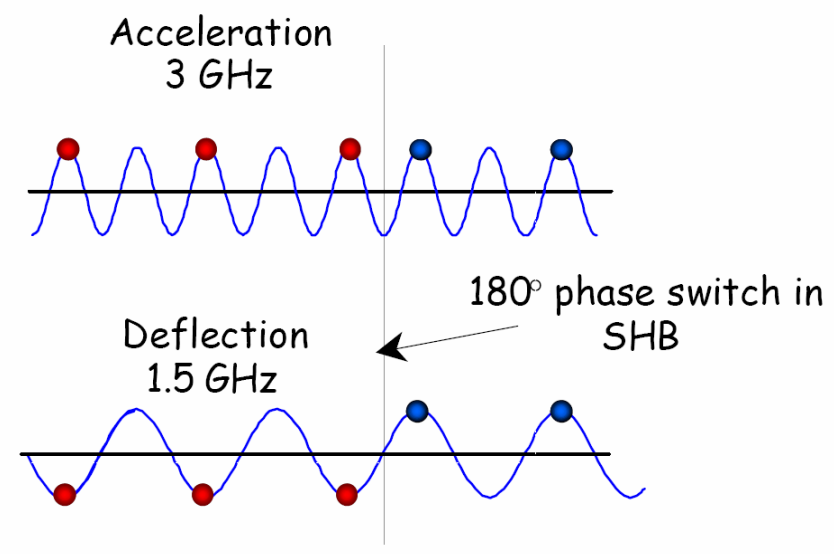

Figure 2. Illustration of required $180^{\circ}$ phase switch between two batches of bunch trains [2].

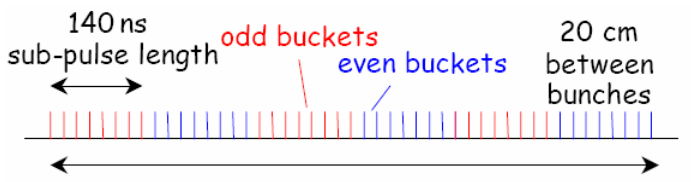

$1.4 \mu$ s train length - 3.5 A current

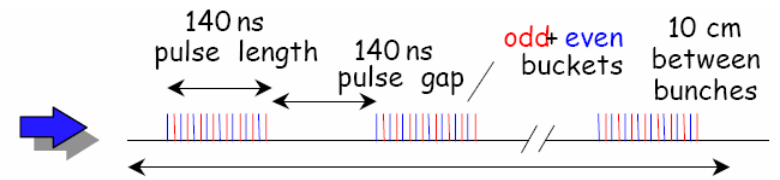

$1.4 \mu$ s train length - 7 A peak current

Figure 3. (x2) Bunch frequency multiplication in the CTF3 Delay Loop [2].

The linac is connected by a transfer line to the Delay Loop [5] where a $1.5 \mathrm{GHz} \mathrm{RF}$ deflector deviates the odd bunch sequences to the left inside the DL and the even ones to the right, figure 1. The DL length is $140 \mathrm{~ns}$ times the velocity of light $\mathrm{c}$ so that after this ring the odd sequence will be recombined with the incoming even sequence to fill the interleaved empty buckets. Precise adjustment of the longitudinal structure can be done with an integrated wiggler. The resulting macro bunch structure at the DL output presents $140 \mathrm{~ns}$ long trains of buckets separated now by $10 \mathrm{~cm}$, followed by $140 \mathrm{~ns}$ long voids.

The timing of the bunches of subsequent batches is adjusted such that they have a phase difference of $180^{\circ}$ with respect to the $1.5 \mathrm{GHz} \mathrm{RF}$ of the deflector, figure 2 .

An overview of the overall bunch combination process is shown in figure 3. The necessary timing is controlled by the sub-harmonic bunchers working at $1.5 \mathrm{GHz}$ in the injector region [6] Every $140 \mathrm{~ns}$ the phase of this RF is changed by 180 degrees. This requires wide band subharmonic buncher structures as well as an RF power source capable of switching phase over a few bunches.

High bandwidth travelling wave tubes (TWT) were chosen as an RF power source 7], which are pulsed giving the sub harmonic bunchers a $4 \mu \mathrm{s} 40 \mathrm{~kW}$ RF pulse with a repetition rate of $5 \mathrm{~Hz}$ [8]. The voltage stability of the power supply is critical in order to achieve a phase variation on the output of the TWT that is less than $2^{\circ}$ for a duration of $1.6 \mu \mathrm{s}$ within the pulse. Since the beam loading is different in each of the three sub harmonic bunchers, the structures are individually detuned.

One possibility to analyze in detail the longitudinal behaviour of the electron bunches in the DL as well as after the recombination process is to use a high speed streak camera with time resolutions down to a few picoseconds, see igure 4] [9]. In order to achieve a good time resolution, first the photons from the radiation to be analyzed are converted to electrons, which are then accelerated and deflected using a time-synchronized, ramped HV electric field. Thus the deflecting filed converts the time information into a spatial information much easier to 


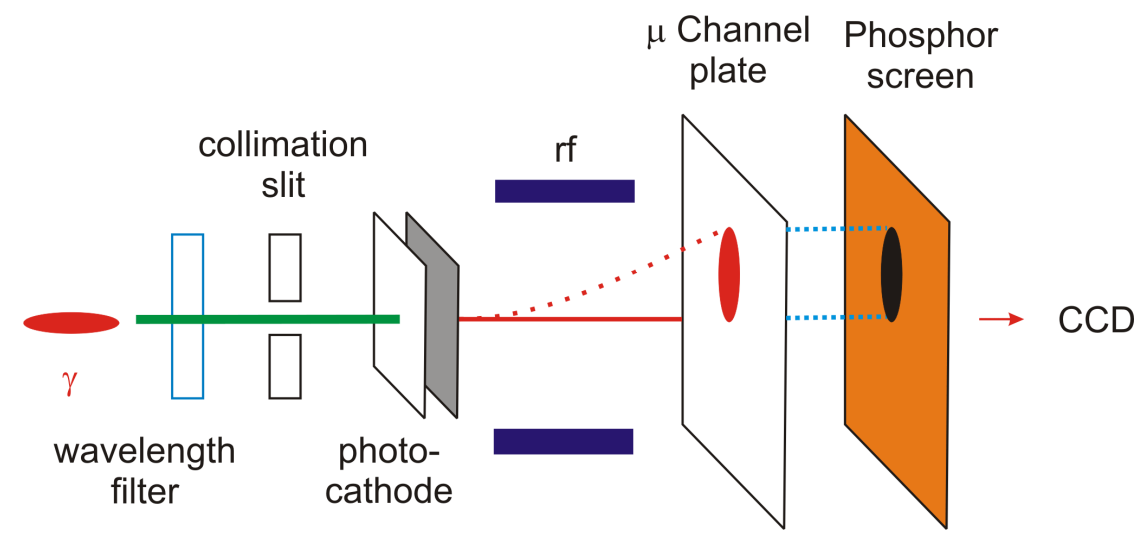

Figure 4. Principle of the streak camera.

analyse. The signal from the electrons is subsequently amplified with a micro channel plate (MCP), converted to photons via a phosphor screen and finally detected using an imager like a CCD array, which converts the light into a voltage signal.

\section{Design considerations}

Optical transition radiation (OTR) [10],[11] and synchrotron radiation (SR) [12],[13] are today established diagnostic techniques that are widely used at accelerator facilities around the world. OTR is an especially interesting candidate since it not only provides a signal in the visible wavelength range that is linear with the bunch charge, but also it offers a spatial resolution down to the $\mu \mathrm{m}$ range and thus covers the requirements at CTF3 [14],[15],[16], Two different kinds of OTR screens are used behind the delay loop while the naturally occurring synchrotron light in the delay loop is exploited for in-ring measurements.

The aim of the here-presented measurement was to study in detail the longitudinal structure of the CTF3 beam and to get information about the different components like the RF deflector or the wiggler. It is a follow-up of a comparable study done at CTF3 (preliminary phase) [17].

Due to the high radiation level in proximity to the beam and the sensitivity of the streak camera, adequate measures have to be taken in order to protect the camera. A measurement close to the accelerator clearly is not feasible. Thus optical lines were designed to guide the light to be observed to the streak camera. It needs to be ensured that a maximum of the created light is collected and projected onto the entrance slit of the streak camera. As will be shown in the following sections the distances that had to be covered by these optical lines reached up to 40 meters and thus required a careful layout.

The design steps can be summarized as follows:

- Transmit the light over large distances using telescopic arrangements, i.e. two identical lenses placed apart two times their focal length.

- Optimize the overall system between collecting, transmitting and demagnifying optics.

- Minimize the number of optical elements in order to maximize light transmission and reduce aberrations. Each lens will reflect about $\sim 10 \%$ of the incident light.

- Optimize the optical resolution. Even though (transverse) aberrations are not the most critical point in the streak camera measurements aimed for at this point in time, one would still like to have a final image where these are minimized. 


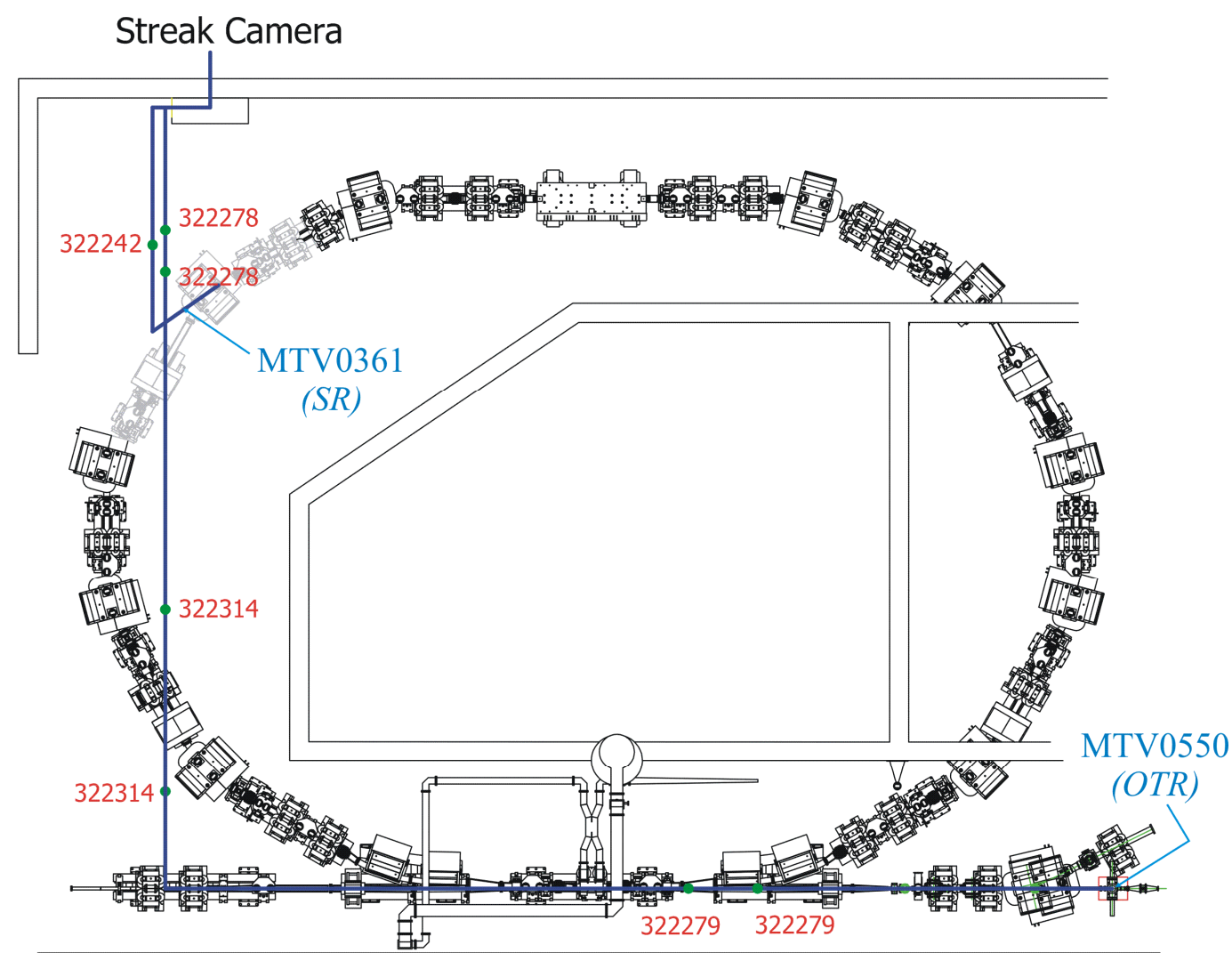

Figure 5. DL with the two optical lines installed in 2005/2006. Numbers correspond to achromatic lenses used. Details are given in the text.

In addition, constraints from the available space in the machine, characteristics of the available lenses and from installations of other diagnostic equipment, influence the final layout of the optics to a high degree. A bird's eye view of the DL with the two optical lines that were installed in 2005/2006 is shown in figure 5.

\section{Light characteristics from MTV0361 and MTV0550}

\subsection{Measurements from MTV0361 using synchrotron light}

In order to find the optimum layout of the optical lines, one needs to know the characteristic parameters of the initial light distribution. Profile measurements based on synchrotron radiation are today well established and used in most high energy storage rings. In the DL, synchrotron light is exploited for measurements at the bending magnets CD.BHE0240, CD.BHF0330 and CD.BHE0360 [18]. While a local CCD camera is installed in the machine in the first two cases, an optical line to the synchrotron light laboratory in building 2002 is used to project light on a CCD camera, the streak camera and an innovative CID camera system [19]. The parameters necessary for the calculation of the SR emission characteristics are summarized in the able 1.

The half opening angle $\theta$ of the light cone emitted at the centre of the bending magnet is given by the relation between the transverse and longitudinal momentum of the circulating electrons [20]

$$
\tan \theta=\frac{p_{y}}{p_{z}}=\frac{p_{0}^{\prime}}{\beta \gamma p_{0}^{\prime}} \approx \frac{1}{\gamma}
$$




\begin{tabular}{|ll|}
\hline Parameter & Value \\
\hline Energy & $150 \mathrm{MeV}$ \\
Relativistic $\gamma$ & 292.5 \\
Current & $5.4 \mathrm{~A}$ \\
Bending Radius & $1.078 \mathrm{~m}$ \\
Bending Angle & $30^{\circ}$ \\
\hline
\end{tabular}

Table 1. Overview of the DL parameters.

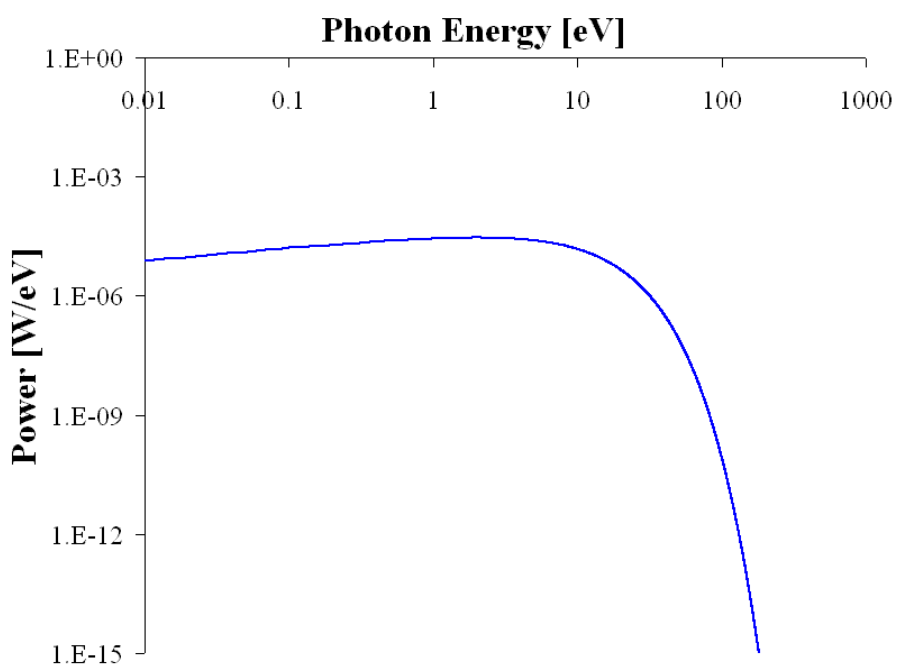

Figure 6. Emitted synchrotron light power as a function of photon energy in the bending magnets of the CTF3 Delay Loop. Beam current: 5.4 A, Energy: $150 \mathrm{MeV}$.

Since $\gamma \gg>1, \tan \theta \approx \theta$ and thus in this particular case $\theta=0.2^{\circ}$. This value is then used in the numerical simulation of the optical line.

A second point of interest when it comes to synchrotron radiation is the emitted power, which can reach enormous values in high energy machines.

It is important to know this parameter to be able to decide e.g. about whether or not cooling of the mirrors is required. As can be seen from figure 6 , the emitted total power does not exceed some $\mathrm{mW}$ and thus does not present a critical factor of this installation.

\subsection{Measurements from MTV0550 using optical transition radiation}

A few meters behind the exit of the Delay Loop, a standard so-called MTV system is installed, see figure 7

Two different screens are mounted on pneumatic arms: A highly reflective silicon screen coated with Aluminium and a Carbon screen for intense, high charge beams. Both are mounted at an angle of $20^{\circ}$ with respect to the beam axis and observation of the emitted light is done via a $40^{\circ}$ view port.

The relative OTR emission intensity of a point-like beam as a function of the emission angle from MTV0550 behind the DL with nominal beam current and at an energy of $E=130 \mathrm{MeV}$ is shown in the following figure 8.

The maximum of the OTR light distribution is found at an emission angle of $\theta=0.2^{\circ}$. Furthermore, it can be seen from the plot that a measurement of the OTR intensity distribution 


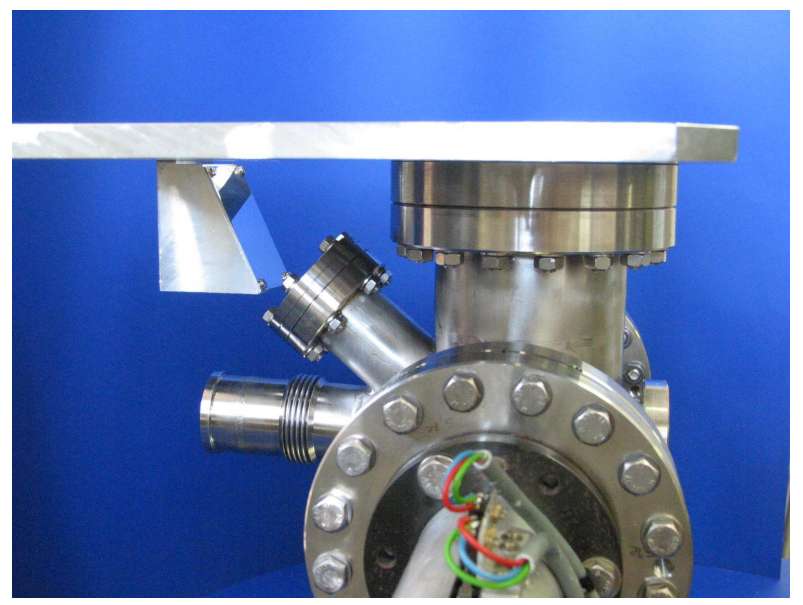

Figure 7. Photograph of standard MTV tank.

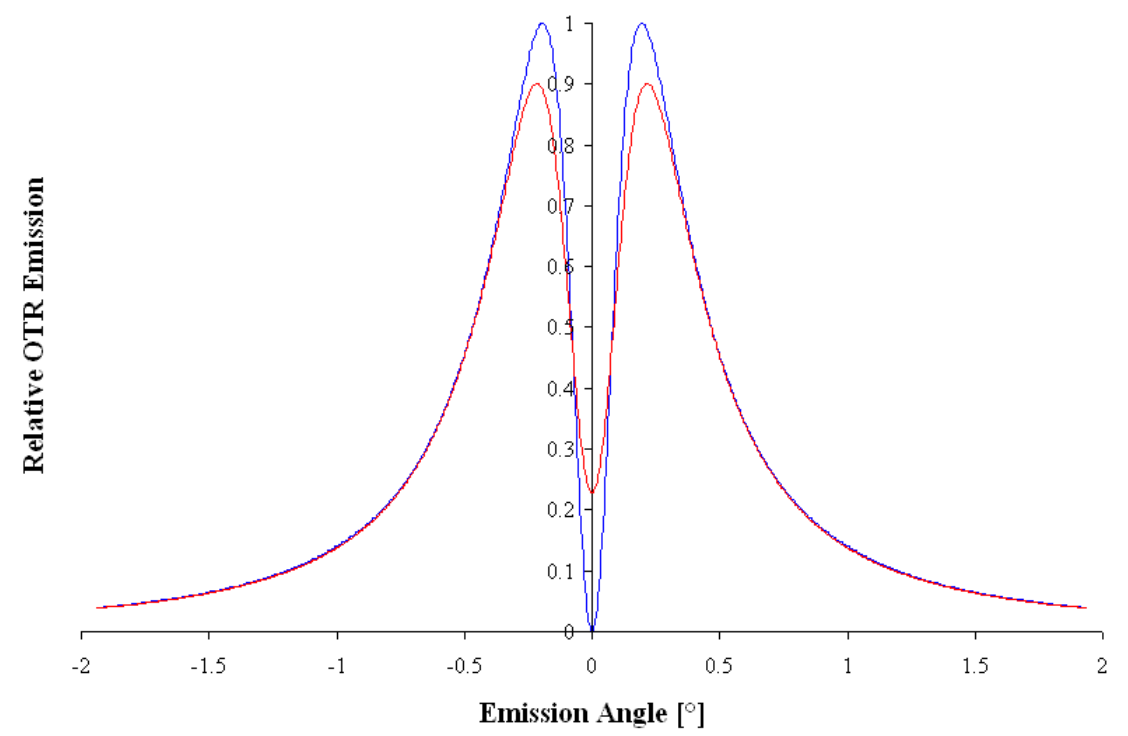

Figure 8. Angular distribution of transition radiation as a function of $\theta$ for a point-like $130 \mathrm{MeV}$ electron beam without $(-)$ and with $(-)$ a beam divergence of $1 \mathrm{mrad}$.

as a function of the emission angle gives direct access to a measurement of the beam divergence, which can even be extended to a single shot emittance measurement [21]. This interesting option might be a choice for CTF3 in the future.

\section{ZEMAX Results}

In order to optimize the overall layout of the optical lines, the computer code ZEMAX 22] was used. It is a comprehensive software tool for optical design and integrates all the features required to conceptualize, design, optimize, analyze, tolerance, and document virtually any optical system. It contains a huge library of lenses from a variety of manufacturers and allows the computation of the quality of the final image.

\subsection{Layout of the optical line from MTV0361}

With a total track length of $11.3 \mathrm{~m}$ between the center of the dipole CD.BHE0360 and the entrance slit of the streak camera, a beam transport by means of only two achromatic lenses and 


\begin{tabular}{|ll|}
\hline Object & Distance $[\mathrm{mm}]$ \\
\hline Light Source $(S R)$ & \\
Mirror & 890 \\
Lens $(f=310 \mathrm{~mm}, \# 322278)$ & 290 \\
Mirror & 560 \\
Lens $(f=1500 \mathrm{~mm}, \# 322242)$ & 1155 \\
Mirror & 2153 \\
Optical Chicane & 6030 \\
Camera & \\
\hline
\end{tabular}

Table 2. List of the elements of the optical line from MTV0361 to the SC lab. Distances are given from one element to the next.
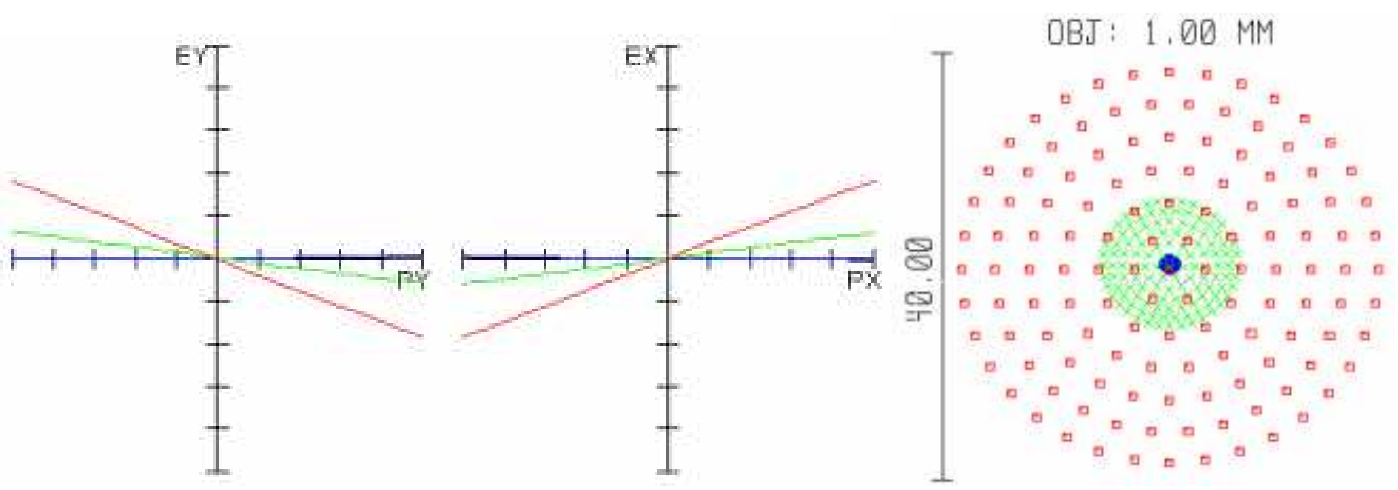

Figure 9. Ray aberrations and spot diagram from MTV0361. The results are shown for a wavelength of $450 \mathrm{~nm}(-), 550 \mathrm{~nm}(-)$ and $650 \mathrm{~nm}(-)$.The vertical scale is given in $\mu \mathrm{m}$.

a final focusing lens right in front of the camera is feasible. All optical manipulations need to be done before the light enters into the final $\sim 4 \mathrm{~m}$ long passage between the buildings 2002 (streak camera lab) and 2003 (DL).

The position of the mirrors is influenced to a high degree by the spatial constraints in the machine while the initial light distribution results from the calculation of the SR emission as presented in the last section. ZEMAX computes the quality of the final image, as well as image magnification and chromatic effects. Furthermore, it provides various tools to optimize the overall setup.

The final result is shown in figure 9. where the ray aberrations and a spot diagram of the final image, i.e. at the position of the CCD or streak camera, are shown. Since this line is also used for transverse beam profile measurements, aberrations need to be reduced as much as possible. The maximum values of the aberrations are around $20 \mu \mathrm{m}$ for an object size of $\pm 15 \mathrm{~mm}$, which corresponds to the size of the entire OTR screen. The characteristic parameters of the line are summarized in the following table 3

\subsection{Layout of the optical line from MTV0550}

As demonstrated before the initial opening angle of the OTR at an energy of $150 \mathrm{MeV}$, as available at MTV0550, is $\theta=0.2^{\circ}$. In contrast to the above described optical line from the DL bending magnet, the distance that needs to be covered stretches out to more than 30 meters. Thus, telescopic arrangements, where two achromatic lenses with identical focal lengths $f$ are 


\begin{tabular}{|ll|}
\hline Description & Value \\
\hline Light Source & Synchroton radiation \\
SR opening angle & $0.2^{\circ}$ \\
Total track length & $11.3 \mathrm{~m}$ \\
Overall magnification & 0.29 \\
Light transmission & $71 \%$ \\
\hline
\end{tabular}

Table 3. Summary of the characteristic parameters of the optical line from MTV0361 to the SC lab.

\begin{tabular}{|ll|}
\hline Object & Distance [mm] \\
\hline Light Source (OTR) & \\
Lens (f=500 mm, \#322279) & 6430 \\
Lens (f=500 mm, \#322279) & 1060 \\
Mirror & 8130 \\
Lens (f=1500 mm, \#322314) & 1550 \\
Lens (f=1500 mm, \#322314) & 2560 \\
Lens (f=310 mm, \#322278) & 2860 \\
Lens (f=310 mm, \#322378) & 650 \\
Mirror & 3310 \\
Optical Chicane & 6030 \\
Camera & \\
\hline
\end{tabular}

Table 4. List of the elements of the optical line from MTV0550 to the SC lab. Distances are given from one element to the next.

placed at a distance $d \approx 2 \cdot f$, had to be used. This configuration guarantees proper light transport over large distances and allows verifying the correct positioning with the help of intermediate image planes. A list of the optical elements used for this line is shown in able 4.

Due to the existing wave guides, vacuum chambers and ventilation tubes around the DL, only a limited number of positions for the lenses were possible and a number of different constraints had to be taken into account throughout the optimization process. In addition, the height of the ceiling in building 2003 changes from $2.50 \mathrm{~m}$ around the RF deflector to $3.0 \mathrm{~m}$ towards the linac and thus a compact optical chicane consisting of two $\mathrm{d}=100 \mathrm{~mm}$ mirrors had to be integrated into the system.

Since the aim of this long line is the analysis of longitudinal beam structure, transverse aberrations in the final image are not as important as in the case of MTV0361. However, one always tries to optimize the quality of the final image to be able to check the correct alignment of the line with the help of intermediate images.

It should be pointed out that the relatively large deviations that can be seen in the spot diagram in figure 10 can be suppressed by introducing a wavelength filter right in front of the streak camera. Whether or not this is possible for a particular measurement depends on the light level needed for a certain time resolution.

A summary of the characteristic parameters of this line is given in the following table 5. 

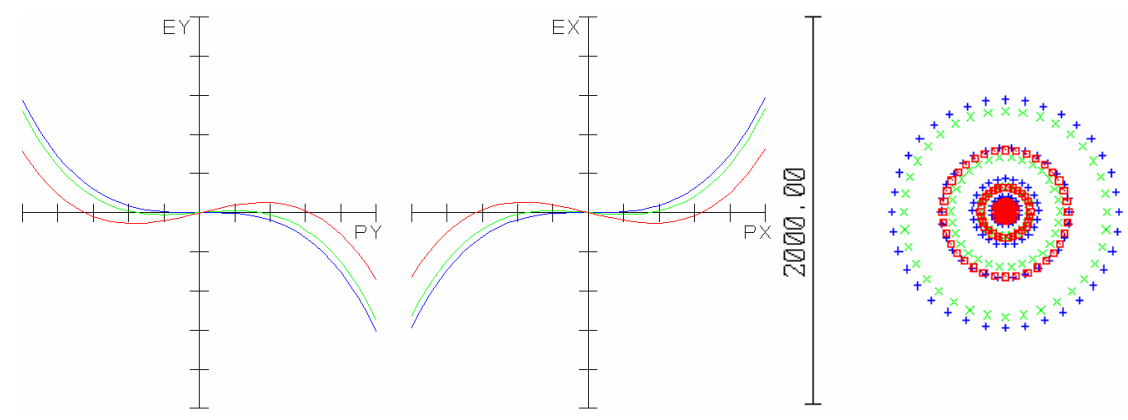

Figure 10. Ray aberrations and spot diagram from MTV0550. The results are shown for a wavelength of $450 \mathrm{~nm}(-), 550 \mathrm{~nm}(-)$ and $650 \mathrm{~nm}(-)$.The vertical scale is given in $\mu \mathrm{m}$.

\begin{tabular}{|ll|}
\hline Description & Value \\
\hline Screen types & Si coated with $\mathrm{Al}$ and C \\
\hline Screen tilt angle & $20^{\circ}$ \\
\hline OTR opening angle & $0.2^{\circ}$ \\
\hline Total track length & $32.6 \mathrm{~m}$ \\
\hline Overall magnification & 0.12 \\
\hline Light transmission & $44 \%$ \\
\hline
\end{tabular}

Table 5. Summary of the characteristic parameters of the optical line from MTV0550 to the SC lab.

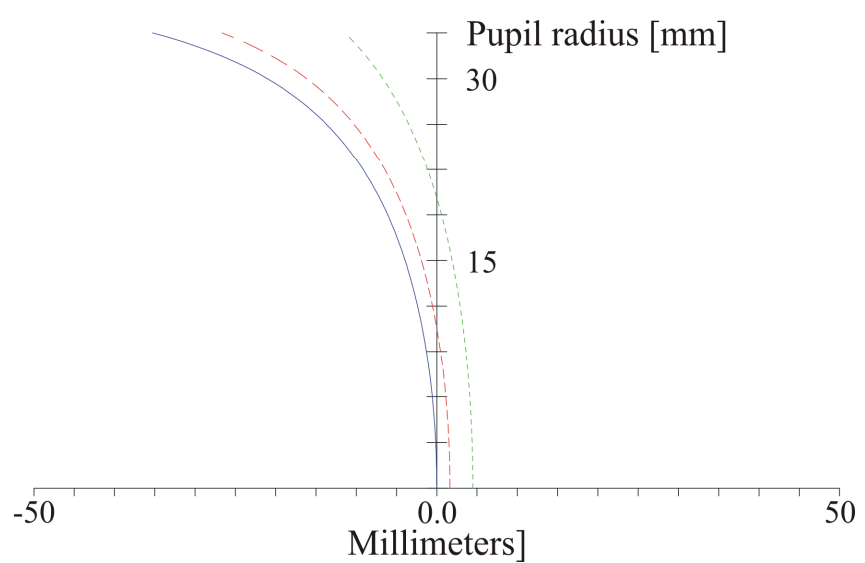

Figure 11. Longitudinal aberrations for three different wavelengths for radial different positions.

With an overall path length of $32.6 \mathrm{~m}$ at a wavelength of $450 \mathrm{~nm}$, the time required for the light to travel the distance from MTV0550 to the streak camera lab is around $113 \mathrm{~ns}$. As the index of refraction of the lenses is a function of the wavelength, the total path lengths is so, too. Figure 11 shows the resulting longitudinal aberrations while table 6 summarizes the resulting spatial and temporal differences. The results clearly indicate that for shortest possible sweep speeds of the available streak camera $(10 \mathrm{ps} / \mathrm{mm})$, wavelength filter will have to be used to suppress longitudinal aberrations that would otherwise dominate the measurements. 


\begin{tabular}{|lll|}
\hline Wavelength $[\mathbf{n m}]$ & $\boldsymbol{\Delta} \mathbf{s}[\mathbf{m m}]$ & $\Delta \mathbf{t}[\mathbf{p s}]$ \\
\hline 450 & reference & 0 \\
550 & -4.48 & -14.9 \\
650 & -2.85 & -9.5 \\
\hline
\end{tabular}

Table 6. Longitudinal aberrations as a function of light wavelength.

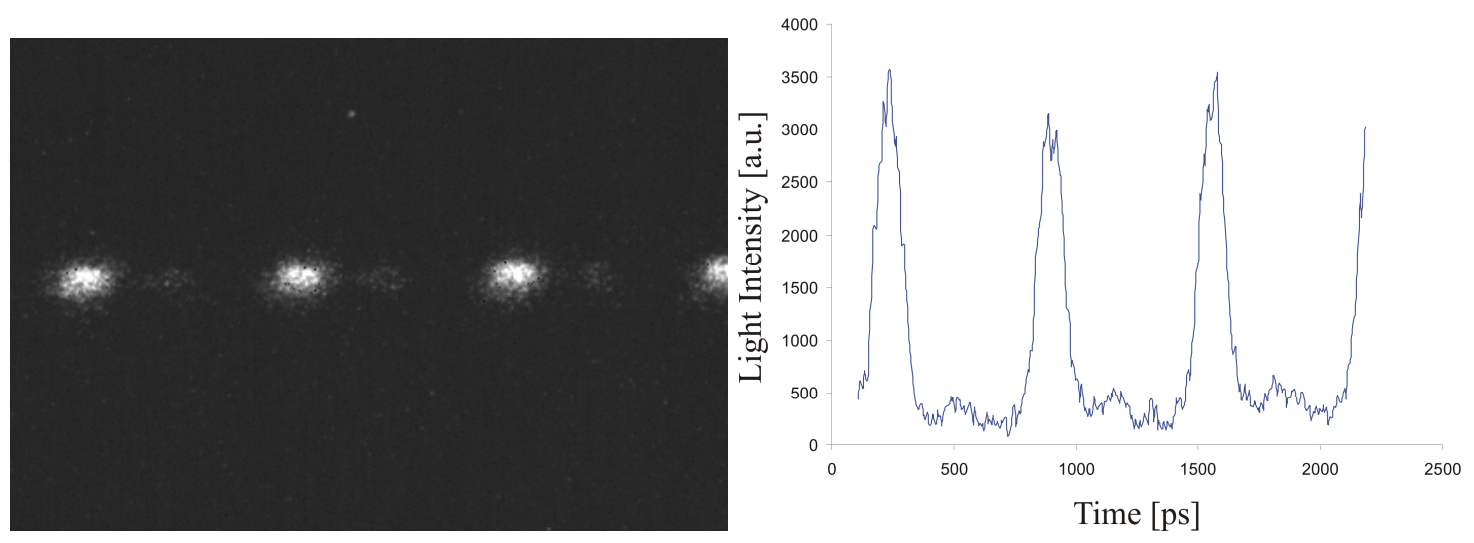

Figure 12. Measured bunch separation in the Delay Loop.

\section{Results from Measurements}

During the last two beam times in 2005 and 2006, the optical lines towards the streak camera were intensely used for monitoring of the longitudinal beam profile and optimization of machine performance. In this section, results from these measurements are shown.

\subsection{Monitoring of the RF bunch combination}

As outlined in the beginning, the CTF3 DL is used for combining two $140 \mathrm{~ns}$ bunch trans with an inter bunch distance of $20 \mathrm{~cm}$ into a single $140 \mathrm{~ns}$ long bunch train where the individual bunches are $10 \mathrm{~cm}$ apart from each other. With one optical line monitoring the light from inside the DL and the other one from behind it, i.e. after bunch combination, the streak camera is the ideal tool for monitoring the longitudinal bunch profiles in both cases.

Measurements of bunch combination were done with a sweep speed of $250 \mathrm{ps} / \mathrm{mm}$ and an
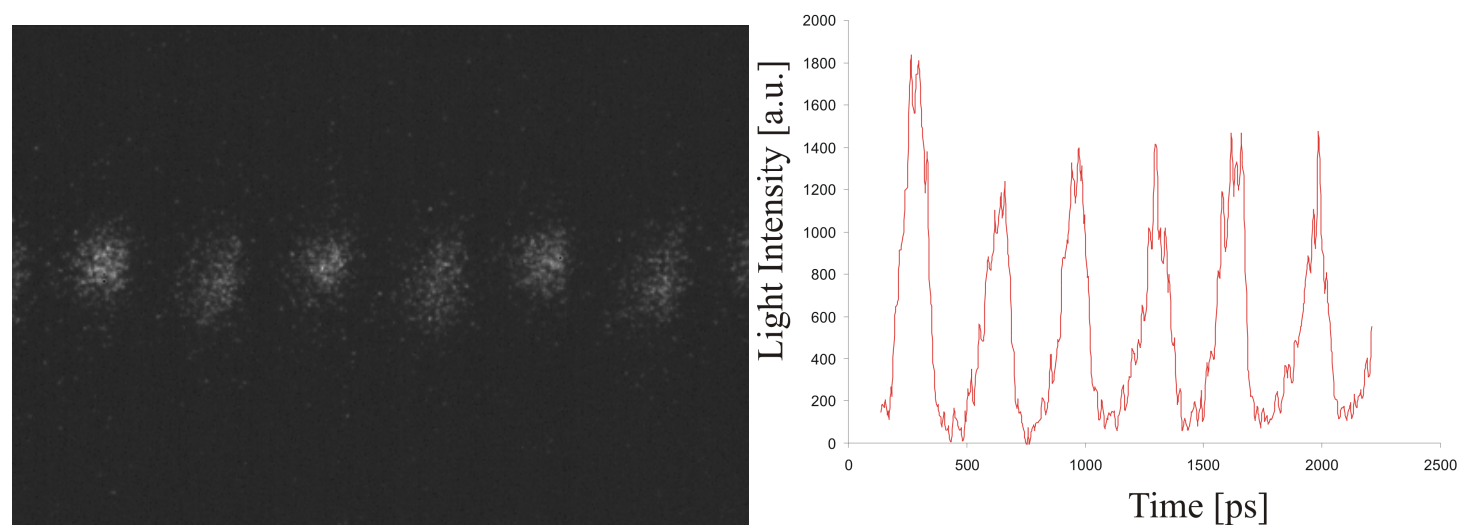

Figure 13. Measured bunch separation after RF recombination. 
image of the measured bunch separation inside the DL is shown in figure 12. The intrinsic resolution of the streak camera used for all the here-presented measurements is $0.25 \mathrm{ps}$.

It can be seen that the train consists not only of the main bunches, but also of weaker satellite bunches of $8.5 \%$ of the main bunches' intensity. The spacing between individual bunches is $666 \mathrm{ps}$ as can be extracted from the beam profile shown on the right of figure 12.

In a second step, the light emerging from the OTR screen at MTV0550 was used to monitor the combined bunch trains after RF deflection, figure 13. Due to the lower transmission
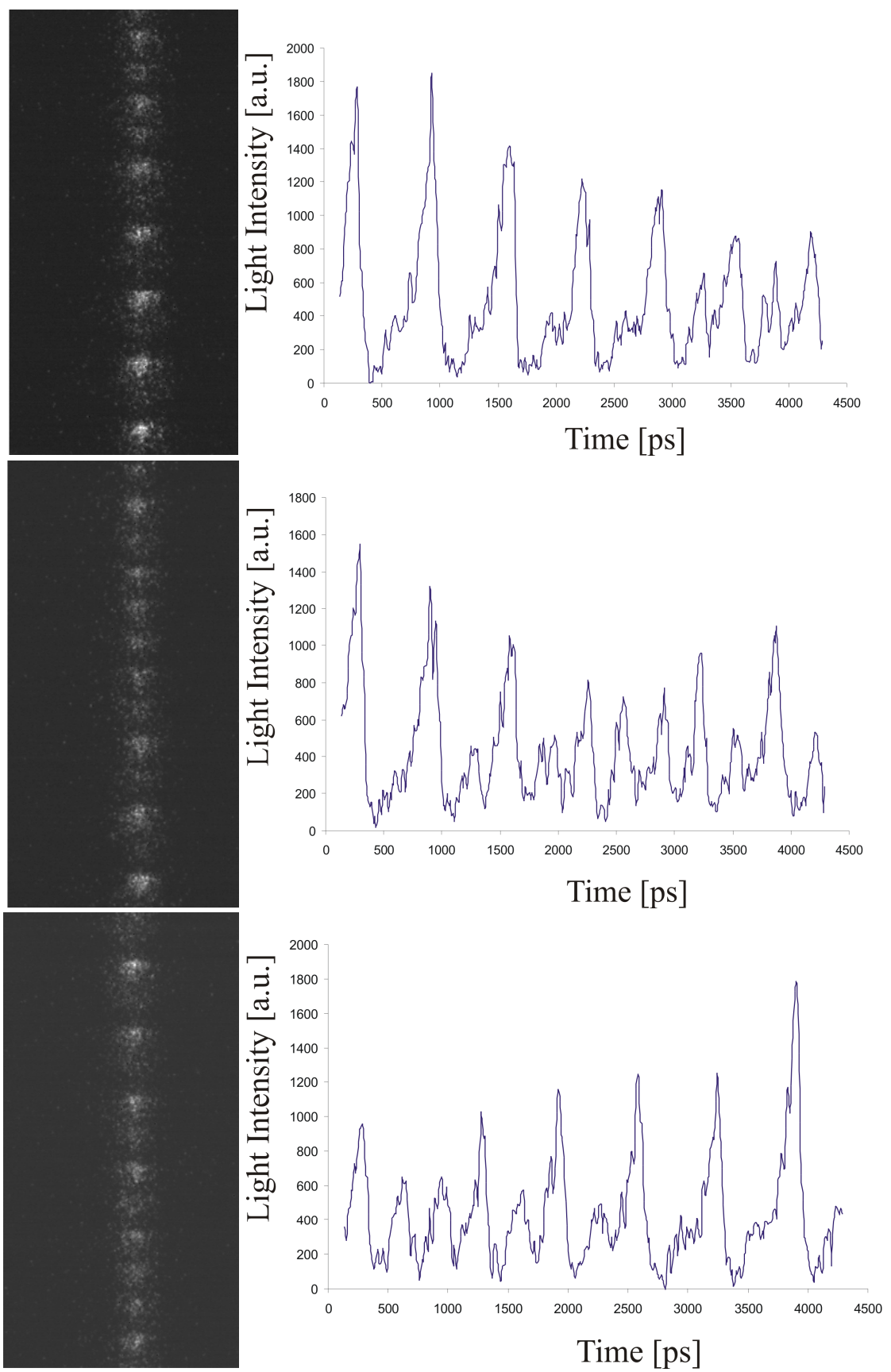

Figure 14. Measured longitudinal beam profiles from MTV0550 without passing the beam through the DL. Images are taken with the trigger at $642 \mathrm{~ns}, 644 \mathrm{~ns}$ and $646 \mathrm{~ns}$. Time is shown on the vertical axis of the streak camera images. 
of the line the signal amplitude is not as high as in the previous case.

With the $1.5 \mathrm{GHz}$ deflector the two bunch trains were successfully combined, resulting in a final bunch spacing of only $10 \mathrm{~cm}$ and double the current of the individual trains. In a future step, these trains will be further combined in the Combiner Ring [23], 24]- another important step towards the demonstration of CLIC feasibility.

\subsection{Monitoring of phase switch using sub-harmonic bunchers}

The CTF3 drive beam generation scheme relies on the use of a fast phase switch of a subharmonic bunching system in order to phase-code the bunches. The amount of charge in unwanted satellite bunches is an important quantity, which must be minimized to avoid unnecessary beam losses and maximize beam transmission. In order to multiply the bunch frequency and the beam current the bunches must be phase coded, meaning that bunches of subsequent batches have a phase difference of $180^{\circ}$ with respect to the $1.5 \mathrm{GHz}$ RF. For that purpose, three $1.5 \mathrm{GHz}$ sub-harmonic bunchers are installed in the accelerator facility. Every $140 \mathrm{~ns}$ the phase of the RF is changed by the necessary $180^{\circ}$. This requires wide band subharmonic buncher structures as well as an RF power source capable of switching over a few bunches.

The streak camera was used to monitor the phase switching process and in particular to determine the time required for the entire switch. The three images in figure 14 were taken from a beam going straight, i.e. without passing through the DL at initial triggers of $642 \mathrm{~ns}, 644 \mathrm{~ns}$ and $646 \mathrm{~ns}$. The overall time required for the switch can then be derived from these measurements to $\tau=5.3 \mathrm{~ns}$, which satisfies the target value.

\subsection{Modification of track length with a wiggler}

For the fine tuning of the machine, a compact wiggler magnet is integrated in the DL. It allows the modification of the ring circumference and thus the optimization of the timing between the two bunch trains to be combined.

In figure 15 the bunch from the DL is slightly off-centre the two bunches from the linac that are at a distance of $666 \mathrm{ps}$. This profile was measured with the wiggler off.

The wiggler was then used for optimization of the bunch spacing and an optimum value

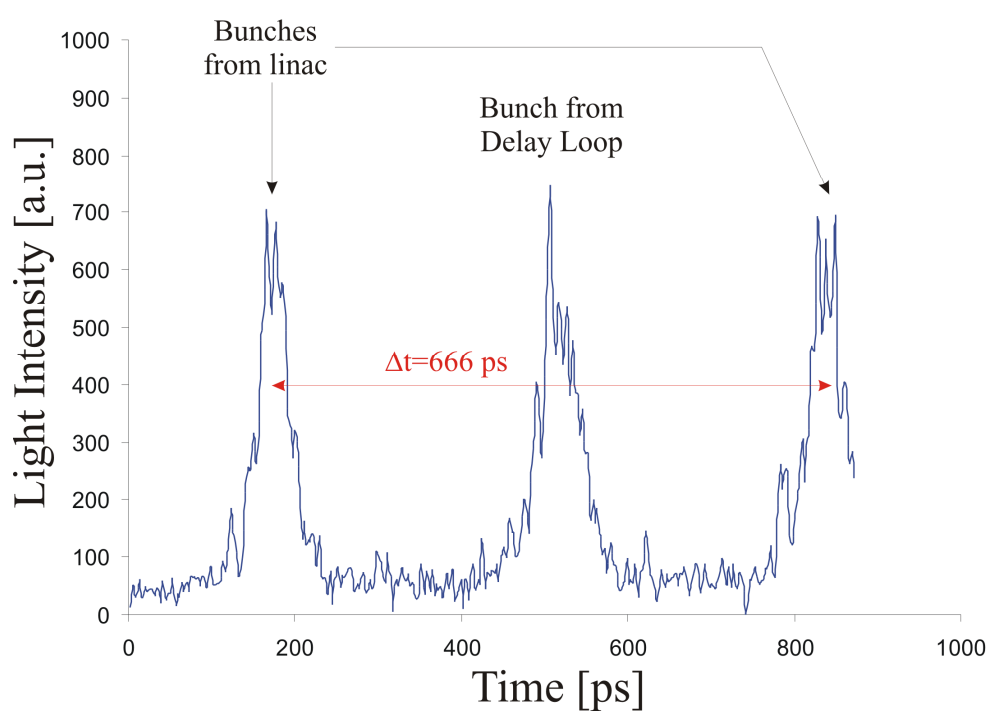

Figure 15. Measured longitudinal beam profile from MTV0550. 


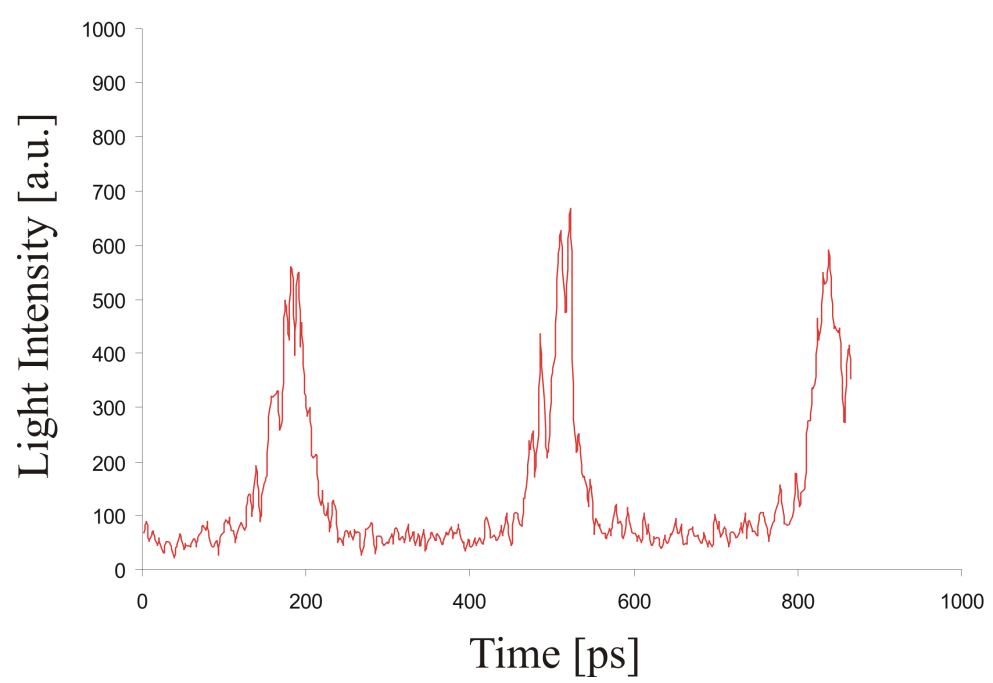

Figure 16. Measured longitudinal beam profile from MTV0550 with an initial offset time of 12 ps.

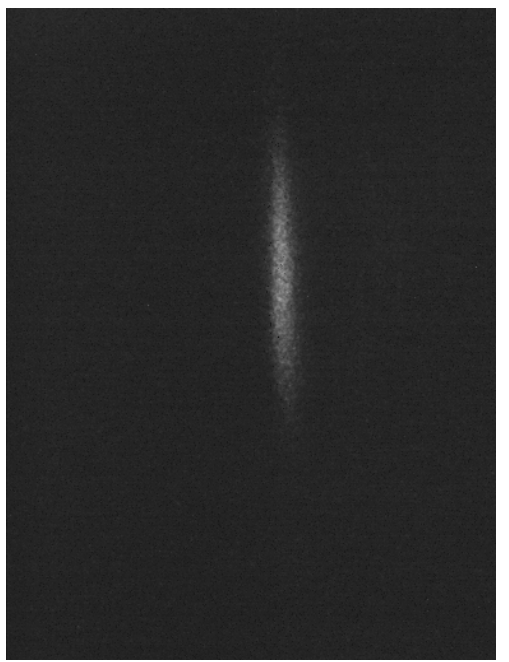

Synchrotron radiation from MTV0361. Measured $\sigma=8.9 \mathrm{ps}$.

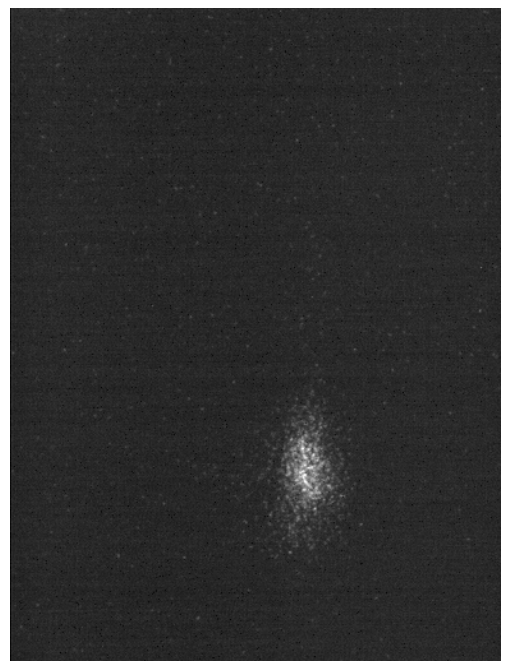

Optical transition radiation from MTV0550. Measured $\sigma=4.5 \mathrm{ps}$.

Figure 17. Measurement of single-bunch longitudinal beam profiles using the highest sweep speed $(10 \mathrm{ps} / \mathrm{mm})$ of the streak camera. Time is shown on the vertical axis.

was found for a current of $62.5 \mathrm{~A}$. Using the streak camera a change by $12 \mathrm{ps}$ - or $3.6 \mathrm{~mm}$ between the bunches was found, corresponding to about half of the total tuning range in the wiggler, figure 16. Sweep speed in this measurement was $100 \mathrm{ps} / \mathrm{mm}$.

\subsection{Bunch Length Measurements with a Streak Camera}

When moving to the highest sweep speed of the streak camera of $10 \mathrm{ps} / \mathrm{mm}$ even bunch length measurements become feasible. Figure 17 shows measured profiles and corresponding $\sigma$ values for bunches in the DL and after the CTF3 linac for two different beam conditions.

This data was compared with calculations based on the optical functions of the machine and an assumed initial bunch distribution and good agreement with the measurements was found. 


\section{Outlook}

The two streak camera lines installed in 2005/2006 allow in-detail monitoring of the longitudinal beam structure at CTF3. This gives access to e.g. bunch length monitoring, monitoring and optimization of the RF recombination as well as tuning of the machine with the integrated wiggler. The streak camera thus provides the ideal tool for measurements on shortest time scales.

In addition to these installations, three more lines will be added in the near future. First, one line from a spectrometer line immediately after the DL will be installed, giving access to fine tuning of the beam energy spread during operation. Furthermore, measurements from light coming from the bending magnets BHF0795 and BHF0750 of the CR will be made available via two additional optical lines. The even longer optical paths in these cases put very high demands on the layout of the optical system. This is true in particular since part of the existing lines will have to be reused and thus a proper matching between the sections needs to be ensured.

Studies with ZEMAX are presently being done and it is foreseen to realize the new installations at the end of 2006.

\section{References}

[1] http://clic-study.web.cern.ch/CLIC-Study

[2] R.W.Assmann et. al., A 3 TeV e $e^{+} e^{-}$Linear Collider Based on CLIC Technology, CERN 2000-008.

[3] G. Geschonke et al., CTF3 Design Report, CERN/PS 2002-008 (RF)

[4] CLIC Study Team, Proposal for Future CLIC Studies and a New CLIC Test Facility (CTF3), CERN/PS 99-047 (LP) and CLIC Note 402 (1999).

[5] F. Sannibale, Driving Beam Delay Loop Design for CTF3, CTF3 Note 023 (2001).

[6] P Urschütz et al., Beam Dynamics and First Operation of the Sub-harmonic Bunching System in the CTF3 Injector, Proc. Europ. Part. Acc. Conf., Edinburgh, Scotland (2006).

[7] G. McMonagle, Operational Performance and Improvements to the RF power sources for the Compact Linear Collider Test Facility (CTF3) at CERN, CLIC note 663 and CERN-Open-2006-30.

[8] G. McMonagle, Technical specication for manufacture of three power supply systems to power TWT's in $C T F$, CERN Invitation to tender IT-3287-AB/CLIC.

[9] M. Uesaka et al., Precise measurement of a subpicosecond electron single bunch by the femtosecond streak camera, Nucl. Instrum. Meth. A406 (1998) 371.

[10] L. Wartski, Etude du rayonnement de transition optique produit par des électrons d'énergie 30 à 70 MeV. Application aux diagnostics de faisceaux de particules chargées, Thesis at the Université de ParisSud (1976).

[11] D.W. Rule and R.B. Fiorito, Imaging Micron Sized Beams with OTR, AIP Conf. Proc. 229 (1991) 315.

[12] C. Bovet et al, The LEP Synchrotron Light Monitors, Proc. IEEE Part. Accel. Conf., San Francisco, USA, (1991) 1160. 
[13] A. Hofmann and F. Meot, Optical resolution of beam cross-section measurements by means of synchrotron radiation, Nucl. Instrum. Meth. A203 (1982) 483.

[14] M. Castellano and V.A. Verzilov, Spatial resolution in optical transition radiation beam diagnostics, PRST-AB 1062801 (1998).

[15] V.A. Lebedev, Diffraction-limited resolution of the optical transition radiation monitor, Nucl. Instrum. Meth. A372 (1996) 344.

[16] X. Artru et al, Resolution power of optical transition radiation: Theoretical considerations, Nucl. Instrum. Meth. B145 (1998) 160.

[17] R. Corsini, et al., Phys. Rev. ST/AB 7 (2004) 040101.

[18] https://edms.cern.ch/file/706436/1/Delay_Loop_2006.ppt

[19] C.P. Welsch et al, Alternative Techniques for Beam Halo Measurements, Meas. Sci. Technol. 17 (2006) 2035.

[20] K. Wille, Physik der Teilchenbeschleuniger und Synchrotronstrahlungsquellen, Teubner (1996).

[21] R.B. Fiorito and D.W. Rule, Optical Transition Radiation Beam Emittance Diagnostics, AIP Conf. Proc. 319 (1994) 21.

[22] http://www.zemax.com

[23] C. Biscari et al, CTF3: Design of Driving Beam Combiner Ring, Proc. $7^{\text {th }}$ European Part. Acc. Conf., Vienna, Austria (2000)

[24] https://edms.cern.ch/file/700154/3/CR_layout.ppt 